\title{
CONSTRUCTION OF HAMILTONIAN-MINIMAL LAGRANGIAN SUBMANIFOLDS IN COMPLEX EUCLIDEAN SPACE
}

\author{
HENRI ANCIAUX AND ILDEFONSO CASTRO
}

\section{IntroduCtion}

A submanifold $L$ of $\mathbb{C}^{n}$ (or more generally of a symplectic manifold $M^{2 n}$ of real dimension $2 n$ ) is said to be Lagrangian if it has dimension $n$ and if the standard symplectic form $\omega$ vanishes on it. This assumption is equivalent to the fact that the standard complex structure $J$ maps the tangent bundle of $L$ onto its normal bundle. A remarkable property of Lagrangian submanifolds of $\mathbb{C}^{n}$ is that those which are in addition minimal, (i.e. critical points for the volume functional) are in fact minimizers in their homology class. The reason is that $\mathbb{C}^{n}$ is endowed with a one-parameter family of calibrations whose calibrated submanifolds are precisely the minimal Lagrangian submanifolds (see [12]). This property does not extend to generic Kähler manifolds, but it does to a certain subclass of them, called Calabi-Yau manifolds (see [12] for a definition). Lagrangian submanifolds of $\mathbb{C}^{n}$ are locally characterized as being the gradient graphs $L:=\left\{(x+i \nabla u(x)), x \in \mathbb{R}^{n}\right\}$ of real-valued functions $u(x)$. In terms of these data, the minimal submanifold equation is (see [12]):

$$
\operatorname{Im} \operatorname{det}_{\mathbb{C}}(I+i \operatorname{Hess} u)=0 .
$$

In the case of dimension 2, it reduces to the famous Monge-Ampère equation.

Besides the classical variational problem of minimizing the volume functional in a homology class (so in particular with respect to compactly supported variations), there is a natural variational problem, first introduced by $\mathrm{Oh}$ (cf [24]), consisting of minimizing the volume with respect to Hamiltonian compactly supported variations. Such variations have the property of preserving the Lagrangian constraint. We shall say that a Lagrangian submanifold is Hamiltonian-minimal ${ }^{1}$

Research partially supported by a MEC-Feder grant MTM2007-61779(Second author).

${ }^{1}$ Some authors use the terminology Hamiltonian stationary 
or H-minimal for short if it is a critical point of the volume for Hamiltonian compactly supported variations. While it is well known that minimal submanifolds are characterized by the vanishing of their mean curvature vector $H=\frac{1}{n} \operatorname{trace} \sigma$, where $\sigma$ is the second fundamental form of $\Phi$, it can be proved that a Lagrangian submanifold of $\mathbb{C}^{n}$ is $\mathrm{H}$-minimal if and only if it satisfies the equation $\operatorname{div} J H=0$, where $\operatorname{div}$ denotes the divergence operator. In particular, Lagrangian submanifolds with parallel mean curvature vector are H-minimal.

The H-minimal equation for a gradient graph is (cf [27]):

$$
\sum_{j=1}^{n} \frac{\partial}{\partial x_{j}} \Delta\left(\frac{\partial u}{\partial x_{j}}\right)=0
$$

where $\Delta$ denotes the Laplacian with respect to the induced metric on $L$. Denoting partial derivatives by subscripts, the latter is given in coordinates by

$$
g_{j k}=\delta_{j k}+\sum_{l=1}^{n} u_{j l} u_{k l} .
$$

In particular the H-minimal equation is of fourth order and its linearization is the bilaplacian equation.

One physical motivation for studying H-minimal Lagrangian submanifold is given by the model of incompressible elasticity: a diffeomorphism $(X(x, y), Y(x, y))$ between two open subsets $U$ and $V$ of $\mathbb{C}$ is incompressible, i.e. $X_{x} Y_{y}-X_{y} Y_{x}=1$, if and only if the graph

$$
L:=\{(x+i y, X(x, y)-i Y(x, y)), x+i y \in U\}
$$

is Lagrangian. Moreover, the diffeomorphism $(X, Y)$ minimizes the functional $\int_{U} \sqrt{1+|\nabla X|^{2}+|\nabla Y|^{2}}$ among incompressible diffeomorphisms if and only if $L$ is H-minimal (cf [28]).

A very important object attached to an oriented Lagrangian submanifold $L$ of $\mathbb{C}^{n}$ is its Lagrangian angle function, which is defined to be the argument of the evaluation of the complex volume form $d z_{1} \wedge \cdots \wedge d z_{n}$. In other words, if $\left(e_{1}, \ldots, e_{n}\right)$ is a tangent frame of $L$, its Lagrangian angle function is given by

$$
\beta=\arg d z_{1} \wedge \cdots \wedge d z_{n}\left(e_{1}, \ldots, e_{n}\right) .
$$

The Lagrangian angle if fundamental in the study of variational problems since it is related to the mean curvature vector of $L$ by the formula $n H=J \nabla \beta$, where $\nabla$ is the gradient for the induced metric. It follows that $L$ is minimal if and only if its Lagrangian angle is equal to a constant $\beta_{0}$. In this case $L$ is in addition calibrated by the $n$-form 
$\operatorname{Re}\left(e^{-i \beta_{0}} d z_{1} \wedge \cdots \wedge d z_{n}\right)$. Moreover, we can deduce a characterization of those Lagrangian submanifolds which are $\mathrm{H}$-minimal in terms of $\beta$ : by the equation $\operatorname{div} J H=-\frac{1}{n} \Delta \beta$, we deduce that a Lagrangian submanifold is $\mathrm{H}$-minimal if and only if its Lagrangian angle is harmonic for the induced metric.

Until recently only very simple examples where known beyond the Cartesian products of $n$ circles $\mathbb{S}^{1}\left(r_{1}\right) \times \ldots \times \mathbb{S}^{1}\left(r_{n}\right) \subset \mathbb{C}^{n}$. After the first description of non-trivial $\mathrm{H}$-minimal tori in [9], the $\mathrm{H}$-minimal cones and tori of $\mathbb{C}^{2}$ where classified respectively in [27] and [16]. Then more examples were discovered in $\mathbb{C}^{2}$ (cf [1]), in $\mathbb{C}^{n}$ (cf [4], [22], [8]) and in $\mathbb{C P}^{n}$ (cf [22], [23], [8]). The goal of the present paper is to describe in a synthetic way a variety of examples of H-minimal Lagrangian immersions in $\mathbb{C}^{n}$, combining in several ways curves in two dimensional space forms and Legendrian immersions in odd dimensional spheres. These constructions appear to be generalizations of examples already discussed in [4], [3] and [8]. In every case we shall characterize the cases in which the submanifold is in addition minimal, or have parallel mean curvature vector. We shall also pay attention to the cases in which we get compact examples. Finally we point out that the importance of H-minimal Lagrangian submanifolds of $\mathbb{C}^{n}$ is emphasized by a recent work of Joyce, Lee and Schoen, where H-minimal Lagrangian submanifolds are constructed in arbitrary symplectic manifolds, starting from a H-minimal Lagrangian submanifold of $\mathbb{C}^{n}$ satisfying some property of rigidity (cf [20]).

The paper is organized as follows: the first section gives some background about curves and Legendrian submanifolds in odd-dimensional spheres; we shall see in particular that the geometry of Legendrian submanifolds is very similar to the one of Lagrangian submanifolds. The next sections are devoted to several constructions of H-minimal Lagrangian submanifolds using, respectively, $n$ planar curves (Section 3), a planar curve and a Legendrian immersion (Section 4), a Lagrangian surface and two Legendrian immersions (Section 5).

\section{BACKGROUND MATERIAL}

2.1. Legendrian immersions. Let $\mathbb{C}^{n}=\left\{\left(z_{1}, \ldots, z_{n}\right), z_{j} \in \mathbb{C}, 1 \leq\right.$ $j \leq n\}$ be the complex Euclidean space of dimension $n$ endowed with the bilinear product

$$
(z, w)=\sum_{j=1}^{n} z_{j} \bar{w}_{j}, \quad \forall z, w \in \mathbb{C}^{n} .
$$


Then $\langle.,\rangle=.\operatorname{Re}(.,$.$) is the Euclidean metric of \mathbb{C}^{n}$ and $\omega=-\operatorname{Im}(.,$. the Kaehler 2-form on $\mathbb{C}^{n}$. Both forms are related by the formula $\omega=\langle J .,$.$\rangle .$

The symplectic form $\omega$ is exact and one primitive of it is the the Liouville 1 -form of $\Lambda$ defined by $2 \Lambda(v)=\langle v, J z\rangle$, for all $v \in T_{z} \mathbb{C}^{n}$, $z \in \mathbb{C}^{n}$.

Next we consider the unit sphere $\mathbb{S}^{2 n-1}:=\left\{z \in \mathbb{C}^{n},\langle z, z\rangle=1\right\}$ and we still denote by $\Lambda$ the restriction to $\mathbb{S}^{2 n-1}$ of the Liouville 1 -form of $\mathbb{C}^{n}$. Hence $\Lambda$ is the contact 1 -form of the canonical Sasakian structure on the sphere $\mathbb{S}^{2 n-1}$. An immersion $\psi$ of an $(n-1)$-dimensional manifold $N$ into $\mathbb{S}^{2 n-1}$ is said to be Legendrian if $\psi^{*} \Lambda \equiv 0$. When it is the case $\psi$ is isotropic in $\mathbb{C}^{n}$, i.e. $\psi^{*} \omega \equiv 0$ and, in particular, the normal bundle of $N$ admits the following decomposition $T^{\perp} N=J(T N) \oplus \operatorname{span}\{J \psi\}$. In other words $\psi$ is horizontal with respect to the Hopf fibration $\Pi$ : $\mathbb{S}^{2 n-1} \rightarrow \mathbb{C} \mathbb{P}^{n-1}$, where $\mathbb{C P}^{n-1}$ denotes the complex projective space with constant holomorphic sectional curvature 4 . Hence $\Pi \circ \psi$ is a Lagrangian immersion of $N$ into $\mathbb{C P}^{n-1}$ and the two immersions $\psi$ and $\Pi \circ \psi$ induce the same metric on $N$. Conversely, given a Lagrangian immersion $\phi$ of a $(n-1)$-dimensional manifold $N$ into $\mathbb{C P}^{n-1}$, there exists a Legendrian immersion $\tilde{\psi}$ from the universal covering $\tilde{N}$ of $N$ into $\mathbb{S}^{2 n-1}$ such that $\phi \circ \tilde{\Pi}=\Pi \circ \tilde{\psi}$, where $\tilde{\Pi}$ is the canonical projection $\tilde{N} \rightarrow N$. The immersion $\tilde{\psi}$, which is unique up to a rotation of the form $e^{i \theta} I d \subset U(n)$, is called the Legendrian lift of $\phi$.

Next we define $\Omega$ to be the complex $(n-1)$-form on $\mathbb{S}^{2 n-1}$ given by

$$
\Omega_{z}\left(v_{1}, \ldots, v_{n-1}\right)=\operatorname{det}_{\mathbb{C}}\left\{z, v_{1}, \ldots, v_{n-1}\right\} .
$$

Given a Legendrian immersion $\psi$ of a manifold $N$ into $\mathbb{S}^{2 n-1}, \psi^{*} \Omega$ is a complex $(n-1)$-form on $N$. Suppose that our Legendrian submanifold $N$ is oriented. We define the map $\beta_{\psi}$ by

$$
e^{i \beta_{\psi}(x)}=\left(\psi^{*} \Omega\right)_{x}\left(e_{1}, \ldots, e_{n-1}\right),
$$

where $\left\{e_{1}, \ldots, e_{n-1}\right\}$ is an oriented orthonormal frame in $T_{x} N$. This $\mathbb{R} / 2 \pi \mathbb{Z}$-valued map is well defined and does not depend on the choice of the frame $\left\{e_{1}, \ldots, e_{n-1}\right\}$. In [8] it is called the Legendrian angle map of $\psi$ and it is proved that

$$
J \nabla \beta_{\psi}=(n-1) H_{\psi},
$$

where $\nabla$ is the gradient with respect to the induced metric in $N$ and $H_{\psi}$ is the mean curvature vector of $\psi$. Hence a Legendrian immersion $\psi$ of an oriented manifold $N$ in $\mathbb{S}^{2 n-1}$ is minimal, i.e. $H_{\psi} \equiv 0$, if and only if the Legendrian angle map $\beta_{\psi}$ of $\psi$ is constant. 
In analogy with the notion of H-minimality for Lagrangian submanifolds, the notion of C-minimality was introduced in [8] as follows: a Legendrian submanifold is said to be contact-minimal (or briefly $C$ minimal) if it is a critical point of the volume functional with respect to compactly supported variations which preserve the contact form. It is easy to prove that a Legendrian immersion $\psi$ is C-minimal if and only if $\operatorname{div} J H_{\psi} \equiv 0$, where div is the divergence operator in $N$. We refer to [8] for the proof of this formula and further details. In particular, minimal Legendrian submanifolds and Legendrian submanifolds with parallel mean curvature vector are C-minimal. A consequence of Equation (1) is that a Legendrian immersion $\psi$ of an oriented manifold $N$ into $\mathbb{S}^{2 n-1}$ is C-minimal if and only if the Legendrian angle $\beta_{\psi}$ of $\psi$ is a harmonic map, i.e. $\Delta \beta_{\psi} \equiv 0$, where $\Delta$ is the Laplacian for the induced metric.

There is a close relationship between between minimal and C-minimal Legendrian submanifolds in odd dimensional spheres and Lagrangian submanifolds in complex projective spaces. More precisely, it is proved in [8] that a Legendrian immersion $\psi$ is minimal (resp. C-minimal) in $\mathbb{S}^{2 n-1}$ if and only if the Lagrangian immersion $\Pi \circ \psi$ is minimal (resp. $\mathrm{H}$-minimal) in $\mathbb{C P}^{n-1}$.

2.2. Planar curves. If $\alpha: I \rightarrow \mathbb{C}^{*}$ is a non-vanishing complex function on an interval $I$ of $\mathbb{R}$, the argument of $\alpha$ is the $\mathbb{R} / 2 \pi \mathbb{Z}$-valued map given by $\alpha=|\alpha| e^{i \arg \alpha}$. It is easy to check that

$$
(\arg \alpha)^{\prime}=\frac{\left\langle\alpha^{\prime}, J \alpha\right\rangle}{|\alpha|^{2}},
$$

and so we deduce that

$$
\left(\arg \alpha^{\prime}\right)^{\prime}=\left|\alpha^{\prime}\right| \kappa_{\alpha},
$$

where $\kappa_{\alpha}$ is the curvature of $\alpha$.

\subsection{Legendrian curves versus spherical and hyperbolic curves.} Let $\mathbb{S}^{3}$ and $\mathbb{H}_{1}^{3}$ denote the unit hypersphere and the unit anti de Sitter space in $\mathbb{C}^{2}$, given respectively by

$\mathbb{S}^{3}=\left\{(z, w) \in \mathbb{C}^{2},|z|^{2}+|w|^{2}=1\right\}, \mathbb{H}_{1}^{3}=\left\{(z, w) \in \mathbb{C}^{2},|z|^{2}-|w|^{2}=-1\right\}$.

Let $\gamma \subset \mathbb{S}^{3}$ and $\alpha \subset \mathbb{H}_{1}^{3}$ two Legendrian curves, both parametrized by arclength. Then they satisfy the following relations:

$$
\begin{gathered}
\left|\gamma_{1}\right|^{2}+\left|\gamma_{2}\right|^{2}=1, \quad\left|\gamma_{1}^{\prime}\right|^{2}+\left|\gamma_{2}^{\prime}\right|^{2}=1, \quad \gamma_{1}^{\prime} \bar{\gamma}_{1}+\gamma_{2}^{\prime} \bar{\gamma}_{2}=0, \\
\left|\alpha_{1}\right|^{2}-\left|\alpha_{2}\right|^{2}=-1, \quad\left|\alpha_{1}^{\prime}\right|^{2}-\left|\alpha_{2}^{\prime}\right|^{2}=1, \quad \alpha_{1}^{\prime} \bar{\alpha}_{1}-\alpha_{2}^{\prime} \bar{\alpha}_{2}=0 .
\end{gathered}
$$


We now detail the special form taken by the Hopf projection $\Pi$ in the case $n=2$, as well as the analogous projection $\mathbb{H}_{1}^{3} \rightarrow \mathbb{H}^{2}(-1 / 2)$.

Let $\mathbb{S}^{2}(1 / 2):=\left\{\left(x_{1}+i x_{2}, x_{3}\right) \in \mathbb{C} \times \mathbb{R}, x_{1}^{2}+x_{2}^{2}+x_{3}^{2}=1 / 4\right\}$ which is the 2 -sphere with radius $1 / 2$ in $\mathbb{R}^{3}$. The Hopf fibration $\Pi: \mathbb{S}^{3} \rightarrow$ $\mathbb{S}^{2}(1 / 2) \equiv \mathbb{C} \mathbb{P}^{1}(4)$ is given by

$$
\Pi(z, w)=\frac{1}{2}\left(2 z \bar{w},|z|^{2}-|w|^{2}\right), \quad(z, w) \in \mathbb{S}^{3} \subset \mathbb{C}^{2} .
$$

For each Legendrian curve $\gamma(s)$ in $\mathbb{S}^{3}$, the projection $\xi=\Pi \circ \gamma$ is a curve in $\mathbb{S}^{2}(1 / 2)$. Conversely, each curve $\xi$ in $\mathbb{S}^{2}(1 / 2)$ gives rise to a horizontal lift $\tilde{\xi}$ in $\mathbb{S}^{3}$ via $\Pi$ which is unique up to a rotation $e^{i \theta} I d \subset U(2), \theta \in \mathbb{R}$.

Since the Hopf fibration $\Pi$ is a Riemannian submersion, each unit speed Legendrian curve $\gamma$ in $\mathbb{S}^{3}$ is projected onto a unit speed curve $\xi$ in $\mathbb{S}^{2}(1 / 2)$ with the same curvature function. In addition:

$$
\left|\gamma_{1}\right|^{2}=\frac{1}{2}+\xi_{3},\left\langle\gamma_{1}^{\prime}, J \gamma_{1}\right\rangle=\left(\xi \times \xi^{\prime}\right)_{3},
$$

where $\times$ denotes the cross product in $\mathbb{R}^{3}$ and $\left(\xi \times \xi^{\prime}\right)_{3}$ is the third coordinate of $\xi \times \xi^{\prime}$ in the 3 -space $\mathbb{R}^{3}$ containing $\mathbb{S}^{2}(1 / 2)$.

Similarly, let $\mathbb{H}^{2}(-1 / 2)=\left\{\left(x_{1}+i x_{2}, x_{3}\right) \in \mathbb{C} \times \mathbb{R}, x_{1}^{2}+x_{2}^{2}-x_{3}^{2}=\right.$ $\left.-1 / 4, x_{3} \geq 1 / 2\right\}$ which is the model of the real hyperbolic plane of curvature -4 . The Hopf fibration $\Pi: \mathbb{H}_{1}^{3} \rightarrow \mathbb{H}^{2}(-1 / 2) \equiv \mathbb{C H}^{1}(-4)$ is then given by

$$
\Pi(z, w)=\frac{1}{2}\left(2 z \bar{w},|z|^{2}+|w|^{2}\right), \quad(z, w) \in \mathbb{H}_{1}^{3} \subset \mathbb{C}^{2} .
$$

Given a Legendrian curve $\alpha(t)$ in $\mathbb{H}_{1}^{3}$, the projection $\eta=\Pi \circ \alpha$ is a curve in $\mathbb{H}^{2}(-1 / 2)$. Conversely, each curve $\eta$ in $\mathbb{H}^{2}(-1 / 2)$ gives rise to a horizontal lift $\tilde{\eta}$ in $\mathbb{H}_{1}^{3}$ via $\Pi$ which is unique up to a rotation of the form $e^{i \theta} I d \subset U(2), \theta \in \mathbb{R}$.

Similarly, if $\alpha$ is a unit speed Legendrian curve in $\mathbb{H}_{1}^{3}$, then the projection $\eta$ is also a unit speed curve in $\mathbb{H}^{2}(-1 / 2)$ with the same curvature function. It follows that

$$
\left|\alpha_{1}\right|^{2}=-\frac{1}{2}+\eta_{3},\left\langle\alpha_{1}^{\prime}, J \alpha_{1}\right\rangle=\left(\eta \times \eta^{\prime}\right)_{3} .
$$

\section{Product of PlanAR CURVES}

The simplest way to obtain Lagrangian submanifolds in $\mathbb{C}^{n}$ is to take the Cartesian product of $n$ planar curves.

Proposition 1. Let $\alpha_{j}$ be $n$ planar regular curves, and denote by $s_{j}$, $1 \leq j \leq n$, the arclength parameter of $\alpha_{j}$. Then the product immersion

$$
\Phi\left(s_{1}, \ldots, s_{n}\right)=\left(\alpha_{1}\left(s_{1}\right), \ldots, \alpha_{n}\left(s_{n}\right)\right)
$$


is a flat Lagrangian immersion whose Lagrangian angle map is given by

$$
\beta_{\Phi}\left(s_{1}, \ldots, s_{n}\right)=\sum_{j=1}^{n} \arg \alpha_{j}^{\prime}\left(s_{j}\right) .
$$

In addition,

$$
D_{\partial_{s_{j}}} \nabla \beta_{\Phi}=\frac{d \kappa_{j}}{d s_{j}} \partial_{s_{j}}
$$

where $\kappa_{j}$ denotes the curvature of the curve $\alpha_{j}, 1 \leq j \leq n$, and $D$ is the Levi-Civita connection of the induced metric, and

$$
\Delta \beta_{\Phi}=\sum_{j=1}^{n} \frac{d \kappa_{j}}{d s_{j}}
$$

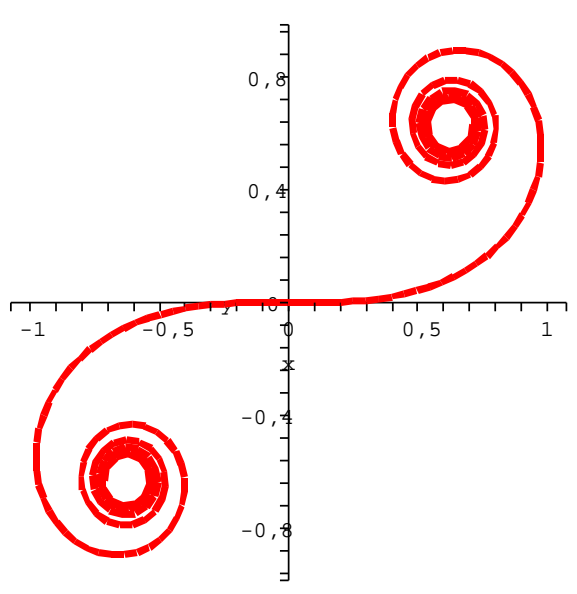

Figure 1. A Cornu spiral (cf Section 3)

Proof. Since the induced metric by the immersion $\Phi$ is flat, using Equation (3) it is straightforward to obtain (6) and hence (7).

Using (3) again, we first observe that $\beta_{\Phi}$ is constant, i.e. $\Phi$ is minimal, if and only if each curve $\alpha_{j}$ is a line. In this case $\Phi$ is totally geodesic.

Moreover, $\Phi$ has parallel mean curvature if and only if each curve $\alpha_{j}$ has constant curvature, i.e. is a straight line or a circle. Finally, $\Delta \beta_{\Phi}=0$, i.e. $\Phi$ is H-minimal if and only if the curvature of each curve $\alpha_{j}$ is a linear function of its arclength parameter, $\kappa_{j}\left(s_{j}\right)=\lambda_{j} s_{j}+\mu_{j}$, and $\sum_{i=1}^{n} \lambda_{j}=0$. Such planar curves are either curves of constant 
curvature (when $\lambda_{j}=0$ ) or special curves known in the literature as Cornu spirals or clotoids (when $\lambda_{j} \neq 0$ ). A Cornu spiral $\alpha$ of parameter $\lambda$ can be parametrized, up to congruences, by

$$
\alpha(s)=\left(\int_{0}^{s} \cos \left(\lambda t^{2} / 2\right) d t, \int_{0}^{s} \sin \left(\lambda t^{2} / 2\right) d t\right) .
$$

The Cornu spirals are bounded but have infinite length. They have two ends that converges to two points of the plane (see Figure 1). In conclusion:

Corollary 1. A product immersion of $n$ planar curves $\alpha_{j}, 1 \leq j \leq n$ have parallel mean curvature if and only if each curve $\alpha_{j}$ is either a circle or a straight line and H-minimal if and only if $(i)$ each curve $\alpha_{j}$ is either a circle, a line, or a Cornu spiral of parametre $\lambda_{j}$, and (ii) $\sum_{j=1}^{n} \lambda_{j}=0$, where we set $\lambda_{j}=0$ if $\alpha_{j}$ is a circle or a line.

Remark 1. We can generalize this construction by considering the product of $n$ Lagrangian immersions into $\mathbb{C}^{m_{j}}, 1 \leq j \leq n$, resulting in a Lagrangian immersion into $\mathbb{C}^{N}$, where $N=\sum_{j=1}^{n} m_{j}$. In the two following sections, we shall consider more elaborate constructions.

\section{Construction with a planar Curve And a Legendrian IMMERSION}

4.1. The construction. We now give a precise description of the geometry of a family of Lagrangian submanifolds in $\mathbb{C}^{n}$ which was first introduced in [26]:

Theorem 1. Let $\alpha: I \rightarrow \mathbb{C}^{*}$ be a regular curve, with arclength parametre $s$ and $\psi$ a Legendrian immersion of an orientable manifold $N$ into $\mathbb{S}^{2 n-1}$. Then the map

$$
\begin{aligned}
& \Phi: I \times N \quad \longrightarrow \quad \mathbb{C}^{n} \\
& (s, x) \longmapsto \alpha(s) \psi(x),
\end{aligned}
$$

is a Lagrangian immersion in $\mathbb{C}^{n}$ with induced metric

$$
\bar{g}=d s^{2}+|\alpha|^{2} g
$$

where $g$ is the induced metric on $N$, and Lagrangian angle map

$$
\beta_{\Phi}(s, x)=G_{\alpha}(s)+\beta_{\psi}(x),
$$

where $G_{\alpha}:=\arg \alpha^{\prime}+(n-1) \arg \alpha$ and $\beta_{\psi}$ is the Legendrian angle map of $\psi$. In addition, the gradient of $\beta_{\Phi}$ is given by the formula:

$$
\nabla_{\bar{g}} \beta_{\Phi}=\left(G_{\alpha}^{\prime} \partial_{s}, \frac{1}{|\alpha|^{2}} \nabla_{g} \beta_{\psi}\right) .
$$


CONSTRUCTION OF H-MINIMAL LAGRANGIAN SUBMANIFOLDS IN $\mathbb{C}^{n} 9$

Moreover, denoting by $\bar{D}$ (resp. by $D)$ the Levi-Civita connection of $\bar{g}$ (resp. of $g$ ), we have

$$
\bar{D}_{\left(\partial_{s}, 0\right)} \nabla_{\bar{g}} \beta_{\Phi}=\left(G_{\alpha}^{\prime \prime} \partial_{s},-\frac{\left\langle\alpha^{\prime}, \alpha\right\rangle}{|\alpha|^{4}} \nabla_{g} \beta_{\psi}\right)
$$

and

$$
\bar{D}_{(0, X)} \nabla_{\bar{g}} \beta_{\Phi}=\left(-\frac{\left\langle\alpha^{\prime}, \alpha\right\rangle}{|\alpha|^{2}} d \beta_{\psi}(X) \partial_{s}, \frac{1}{|\alpha|^{2}} D_{X} \nabla_{g} \beta_{\psi}+G_{\alpha}^{\prime} \frac{\left\langle\alpha^{\prime}, \alpha\right\rangle}{|\alpha|^{2}} X\right),
$$

where $X$ is a vector field on $N$.

Finally, the Laplacian of $\beta_{\Phi}$ is given by the formula:

$$
\Delta_{\bar{g}} \beta_{\Phi}=\frac{1}{|\alpha|^{n-1}} \frac{d}{d s}\left(|\alpha|^{n-1} G_{\alpha}^{\prime}\right)+\frac{1}{|\alpha|^{2}} \Delta_{g} \beta_{\psi} .
$$

Proof. We consider local coordinates $\left(x_{1}, \ldots, x_{n-1}\right)$ on $N$, so that we get local coordinates $\left(x_{0}=s, x_{1}, \ldots, x_{n-1}\right)$ on $I \times N$. We calculate the first derivatives of the immersion:

$$
\frac{\partial \Phi}{\partial x_{0}}=\alpha^{\prime} \psi, \quad \frac{\partial \Phi}{\partial x_{j}}=\alpha \frac{\partial \psi}{\partial x_{j}}, \quad 1 \leq j \leq n-1 .
$$

Calculating the Hermitian products of pairs of such first derivatives and using the Legendrian property of $\psi$ shows that the immersion $\Phi$ is Lagrangian. Moreover, denoting by $\left(g_{j k}\right)_{1 \leq j, k \leq n-1}$ the coefficients of the metric $g$, it follows that the coefficients of the metric $\bar{g}$ are given by

$$
\bar{g}_{j k}=|\alpha|^{2} g_{j k} \quad \bar{g}_{0 j}=0 \quad \bar{g}_{00}=1,
$$

and the inverse matrix of $\bar{g}$ by

$$
\bar{g}^{j k}=|\alpha|^{-2} g^{j k} \quad \bar{g}^{0 j}=0 \quad \bar{g}^{00}=1 .
$$

In particular we obtain the formula

$$
\bar{g}=d s^{2}+|\alpha|^{2} g .
$$

Next we compute the Lagrangian angle of $\Phi$ :

$$
\begin{gathered}
\beta_{\Phi}=\arg \operatorname{det}_{\mathbb{C}}\left(\frac{\partial \Phi}{\partial x_{0}}, \ldots, \frac{\partial \Phi}{\partial x_{n-1}}\right) \\
=\arg \left(\alpha^{\prime} \alpha^{n-1} \operatorname{det}_{\mathbb{C}}\left(\psi, \frac{\partial \psi}{\partial x_{1}}, \ldots, \frac{\partial \psi}{\partial x_{n-1}}\right)\right) \\
=\arg \alpha^{\prime}+(n-1) \arg \alpha+\beta_{\psi} .
\end{gathered}
$$


We deduce the following expression of the gradient of $\beta_{\Phi}$ :

$$
\begin{gathered}
\nabla_{\bar{g}} \beta_{\Phi}=\sum_{a, b=0}^{n-1} \bar{g}^{a b} \frac{\partial \beta_{\Phi}}{\partial x_{b}} \partial_{x_{a}}=\bar{g}^{00} G_{\alpha}^{\prime}\left(\partial_{s}, 0\right)+\frac{1}{|\alpha|^{2}} \sum_{j, k=1}^{n-1} g^{j k} \frac{\partial \beta_{\psi}}{\partial x_{k}}\left(0, \partial_{x_{j}}\right) \\
=G_{\alpha}^{\prime}\left(\partial_{s}, 0\right)+\frac{1}{|\alpha|^{2}}\left(0, \nabla_{g} \beta_{\psi}\right) .
\end{gathered}
$$

Next, a straightforward computation gives

$$
\bar{D}_{\partial_{s}} \partial_{s}=0 \quad \bar{D}_{\partial_{x_{j}}} \partial_{s}=\bar{D}_{\partial_{s}} \partial_{x_{j}}=\frac{\left\langle\alpha^{\prime}, \alpha\right\rangle}{|\alpha|^{2}} \partial_{x_{j}}
$$

and

It follows that

$$
\bar{D}_{\partial_{x_{j}}} \partial_{x_{k}}=-\left\langle\alpha^{\prime}, \alpha\right\rangle g_{j k} \partial_{s}+D_{\partial_{x_{j}}} \partial_{x_{k}}
$$

$$
\bar{D}_{\partial_{s}} \nabla_{\bar{g}} \beta_{\Phi}=\left(G_{\alpha}^{\prime \prime} \partial_{s}, 0\right)
$$

and

$$
\bar{D}_{\partial_{x_{j}}} \nabla_{\bar{g}} \beta_{\Phi}=\left(-\frac{\left\langle\alpha^{\prime}, \alpha\right\rangle}{|\alpha|^{2}} \frac{\partial \beta_{\psi}}{\partial x_{j}} \partial_{s}, \frac{1}{|\alpha|^{2}} D_{\partial_{x_{j}}} \nabla_{g} \beta_{\psi}+G_{\alpha}^{\prime} \frac{\left\langle\alpha^{\prime}, \alpha\right\rangle}{|\alpha|^{2}} \partial_{x_{j}}\right) .
$$

This implies Equation (11) and it remains to compute the Laplacian of $\beta_{\Phi}$ :

$$
\begin{gathered}
\Delta_{\bar{g}} \beta_{\Phi}=\sum_{j, k=0}^{n-1} \bar{g}\left(\bar{D}_{\partial_{x_{j}}} \nabla_{\bar{g}} \beta_{\Phi}, \partial_{x_{k}}\right) \bar{g}^{j k} \\
=G_{\alpha}^{\prime \prime}+\sum_{j, k=1}^{n-1} g\left(\frac{1}{|\alpha|^{2}} D_{\partial_{x_{j}}} \nabla_{g} \beta_{\psi}, \partial_{x_{k}}\right) g^{j k}+\sum_{j, k=1}^{n-1} G_{\alpha}^{\prime} \frac{\left\langle\alpha^{\prime}, \alpha\right\rangle}{|\alpha|^{2}} g\left(\partial_{x_{j}}, \partial_{x_{k}}\right) g^{j k} \\
=G_{\alpha}^{\prime \prime}+\frac{1}{|\alpha|^{2}} \Delta_{g} \beta_{\psi}+(n-1) G_{\alpha}^{\prime} \frac{\left\langle\alpha^{\prime}, \alpha\right\rangle}{|\alpha|^{2}} .
\end{gathered}
$$

Finally, we observe that

$$
\frac{1}{|\alpha|^{n-1}} \frac{d}{d s}\left(|\alpha|^{n-1} G_{\alpha}^{\prime}\right)=G_{\alpha}^{\prime \prime}+(n-1) G_{\alpha}^{\prime} \frac{\left\langle\alpha^{\prime}, \alpha\right\rangle}{|\alpha|^{2}}
$$

and the proof is complete.

Remark 2. If $\alpha$ is a straight line passing through the origin, the immersion becomes $\Phi: \mathbb{R} \times N \longrightarrow \mathbb{C}^{n},(s, x) \rightarrow s \psi(x)$. Hence we obtain the cone $C(\psi)$ with link $\psi$. It is clear that $C(\psi)$ is a Lagrangian immersion with a singularity at $s=0$. In the general case $\Phi$ has singularities at the points $(s, x) \in I \times N$ where $\alpha$ vanishes. Since in the case of a cone $\beta_{C(\psi)}=\beta_{\psi}$, we deduce $C(\psi)$ is minimal (resp. H-minimal) if and only if $\psi$ is minimal (resp. C-minimal); this result was used in [13] and [14]. 
If we take $\alpha(s)=e^{i s}$, Theorem 1 gives a family of submanifolds which generalizes to higher dimension the Hopf cylinders considered in [25].

If $\psi$ is chosen to be the totally geodesic Legendrian embedding $\psi(x)=x$ of $\mathbb{S}^{n-1}$ into $\mathbb{S}^{2 n-1}$, we are in the case of the Lagrangian submanifolds which are invariant under the standard action of $S O(n)$ on $\mathbb{C}^{n}$ (see [12], [4]).

The quantity $A_{\alpha}:=|\alpha|^{n-1} G_{\alpha}^{\prime}$ appearing in the expressions of the gradient and the Laplacian of $\beta_{\Phi}$ enjoys a geometric interpretation. In fact, a simple calculation using Equation (3) yields

$$
A_{\alpha}=|\alpha|^{2 n-2} \kappa_{\alpha^{n}} .
$$

where $\kappa_{\alpha^{n}}$ is the curvature of the planar curve $\alpha^{n}$. From Equation (9) and the formula $n H=J \nabla \beta$, it follows that the immersion $\Phi$ constructed in Theorem 1 is minimal if and only if $\psi$ is minimal and $\alpha^{n}$ has curvature zero. This fact has been used in [10], [13] or [18]. If $\alpha$ is not a straight line, it can be parametrized by $\alpha_{c}(t)=\sqrt[2 n]{t^{2}+c^{2}} e^{i \frac{\arctan t}{n c}}$ and the corresponding minimal Lagrangian submanifolds were constructed in [10], (Remark 1), [13], (Theorem A) and [18] (Theorem 6.4). We refer to [11] for the description of some other examples of minimal Lagrangian submanifolds in $\mathbb{C}^{n}$ using this method.

Next we give a characterization of those immersions described in Theorem 1 which have parallel mean curvature vector or are compact and H-minimal:

Corollary 2. The Lagrangian immersion

$$
\begin{aligned}
& \Phi: I \times N \rightarrow \mathbb{C}^{n} \\
& (s, x) \mapsto \alpha(s) \psi(x),
\end{aligned}
$$

where $\alpha$ is a planar curve which does not vanish and $\psi$ is a Legendrian immersion of an orientable manifold $N$ into $\mathbb{S}^{2 n-1}$, has parallel mean curvature vector if and only if

- either $(i)$ it is minimal; in this case the curve $\alpha$ is such that the quantity $A_{\alpha}:=|\alpha|^{2 n-2} \kappa_{\alpha^{n}}$, where $\kappa_{\alpha^{n}}$ is the curvature of $\alpha^{n}$, vanishes and the immersion $\psi$ is minimal;

- or (ii) the curve $\alpha$ is a circle centered at the origin and the immersion $\psi$ has parallel mean curvature vector.

Moreover, if $N$ is compact, the immersion $\Phi$ is H-minimal if and only if the curve $\alpha$ is such that $A_{\alpha}$ is constant and the immersion $\psi$ is C-minimal.

Proof. The first claim follows easily from Equations (10) and (11). To prove the second claim, we observe that if $\Phi$ is H-minimal, i.e. $\Delta_{\bar{g}} \beta_{\Phi}$ 
vanishes, then $\Delta_{g} \beta_{\psi}$ must be constant by Equation (12). Moreover, if $N$ is compact, $\int_{N} \Delta_{g} \beta_{\psi}$ vanishes so this constant must be zero (so in particular $\psi$ is C-minimal). Thus, by Equation (12) again, $A_{\alpha}$ must be constant.

In the next two sections we shall describe in greater detail respectively the planar curves $\alpha$ and the Legendrian immersions $\psi$ satisfying the conditions given in Corollary 2 to get H-minimal Lagrangian submanifolds in $\mathbb{C}^{n}$.

4.2. Planar curves satisfying $A_{\alpha}=|\alpha|^{2 n-2} \kappa_{\alpha^{n}}$ is a non null constant. The simplest curves verifying this condition are the circles centered at the origin, $\alpha(s)=R e^{i s / R}, R>0$. By Corollary 2, it provides examples of Lagrangian H-minimal immersions of $\mathbb{S}^{1} \times N^{n-1}$ in $\mathbb{C}^{n}$. In particular, taking $\psi(x)=x, x \in \mathbb{S}^{2 n-1}$, we recover Example 2.10 in [20].

Since the curvature of a curve is changed under a scaling according to the law $\kappa_{\lambda \alpha}=\kappa_{\alpha} / \lambda$, we deduce that $A_{\lambda \alpha}=\lambda^{n-2} A_{\alpha}$. Thus, when $n=2$ this quantity $|\alpha|^{2} \kappa_{\alpha^{2}}$ is invariant under dilations; on the contrary, when $n>2$ we can normalize $A_{\alpha}=1$ by rescaling the curve. A qualitative study of the curves $\alpha$ solutions of $|\alpha|^{2 n-2} \kappa_{\alpha^{n}}=$ constant has been done for $n=2$ in [3], Section 4.1 p. 15 and the case $n>2$ has been treated in [4], Section 5, p. 1204. In both cases the purpose was the classification of H-minimal Lagrangian submanifolds in $\mathbb{C}^{n}$ foliated by $(n-1)$-dimensional spheres. We give here a brief description of these curves (see Figures 2,3,4 and 5):

Case $n=2$. Beyond the circles centered at the origin, the solutions of the equation $|\alpha|^{2} \kappa_{\alpha^{2}}=$ constant belong to one of the following families:

- A two-parameter family of non-embedded curves, including a countable family of closed curves that we shall denote by $\alpha_{p}, p \in$ $\mathbb{N}$. The other ones are not properly embedded;

- A one-parameter family of unbounded, non-embedded curves.

Case $n>2$. Beyond the circles centered at the origin, the solutions of the equation $|\alpha|^{2 n-2} \kappa_{\alpha^{n}}=$ constant belong to one of the following families:

- A one-parameter family of non-embedded curves, including a countable family of closed curves that we shall denote by $\alpha_{p}, p \in$ $\mathbb{N}$. The other ones are not properly embedded;

- A one-parameter family of unbounded curves, some of them being embedded and other ones not; 


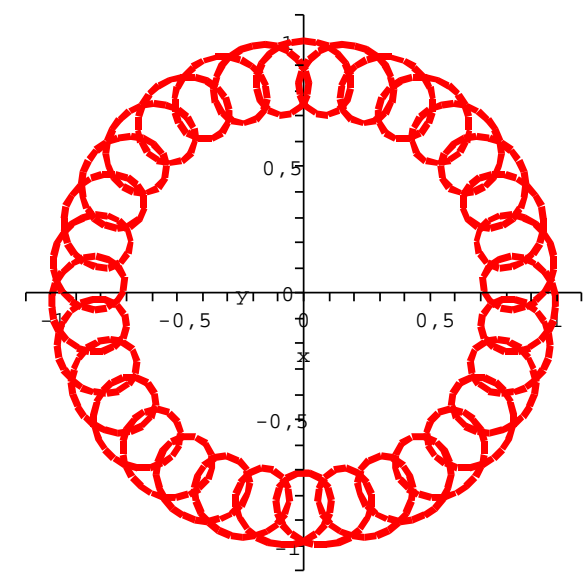

Figure 2. A closed curve $\alpha_{p}$ with $A_{\alpha}=5, n=2$

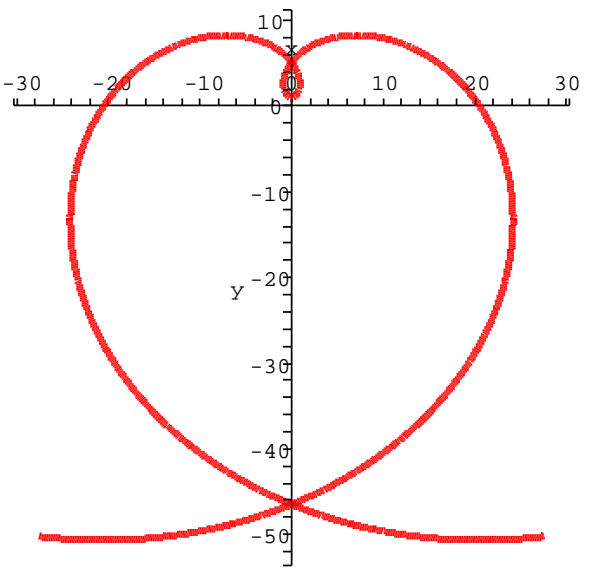

FiguRE 3. An unbounded curve with $A_{\alpha}=1, n=2$

- A one-parameter family of curves which are not properly embedded, with a spiraling end asymptotic to a circle centered at the origin;

- A one-parameter family of curves with two spiraling ends asymptotic to a circle centered at the origin; 


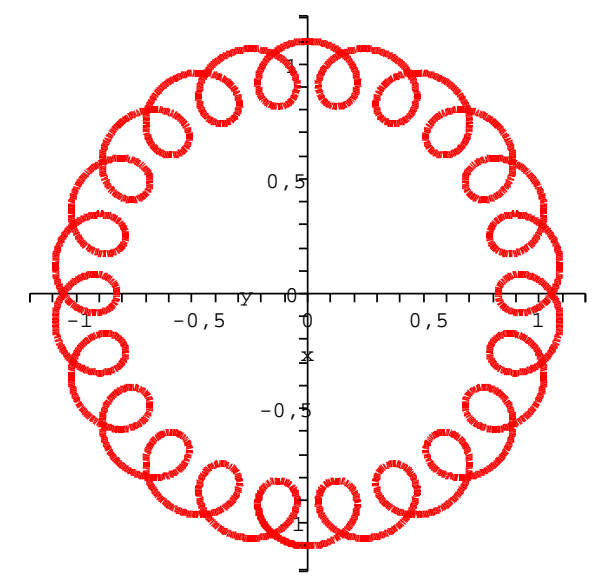

Figure 4 . A closed curve $\alpha_{p}$ with $n=3$

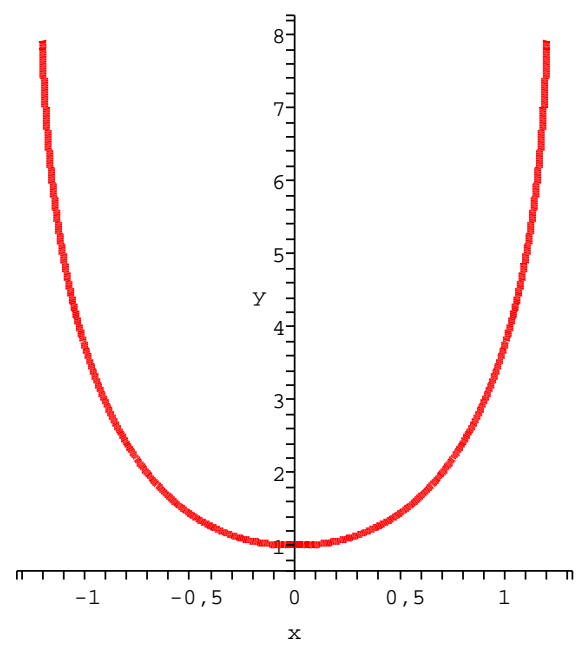

FiguRE 5. An unbounded curve with $n=3$

- Moreover, in the case of dimension $n=3$, the straight lines (not passing through the origin) are solutions.

\subsection{C-minimal Legendrian submanifolds in odd dimensional} spheres. We now describe several examples of C-minimal Legendrian immersions into $\mathbb{S}^{2 n-1}$, making special emphasis in the compact case. We distinguish several cases according to the values of $n$. 
4.3.1. Case $n=2$. In this case, the Laplacian operator on a Legendrian curve $\gamma$ in $\mathbb{S}^{3}$ is simply the second derivative with respect to the arclength parameter $s$. Since $\frac{d \beta_{\gamma}}{d s}=k_{\gamma}$, we deduce $\gamma$ is C-minimal if and only if it has constant curvature $k_{\gamma}=c$.

Such curves take the following explicit form (see [7]):

$$
\gamma(s)=e^{i\left(c+\sqrt{c^{2}+4}\right) s / 2} A_{1}+e^{i\left(c-\sqrt{c^{2}+4}\right) s / 2} B_{1},
$$

for suitable constants $A_{1}, B_{1} \in \mathbb{C}^{2}$. Since $\Phi(t, s)=\alpha(t) \gamma(s)$, up to rotations it is enough to consider the Legendrian curves parametrized by

$$
\gamma_{\varphi}(s)=\left(\cos \varphi e^{i \tan \varphi s}, \sin \varphi e^{-i \cot \varphi s}\right), \varphi \in(0, \pi / 2),
$$

where $\pi / 2-2 \varphi$ is the latitude of the parallel $\pi \circ \gamma_{\varphi}$. It is straightforward to check that the Legendrian angle of $\gamma_{\varphi}$ is

$$
\beta_{\gamma_{\varphi}}=-\pi / 2+(\tan \varphi-\cot \varphi) s,
$$

hence it has constant curvature $c=\tan \varphi-\cot \varphi$. Moreover the curve is closed if and only if $\tan ^{2} \varphi=p / q \in \mathbb{Q}$. These curves are the links of the cones whose Hamiltonian stability is studied in Theorem 7.1 of $[27]$.

4.3.2. Case $n=3$. There is a Legendrian immersion of the torus $\mathbb{S}^{1} \times \mathbb{S}^{1}$ into $\mathbb{S}^{5}$ which is flat and minimal: $\psi(s, t):=\left(e^{i s}, e^{i t}, e^{-i(s+t)}\right)$. Besides this trivial example, there is a growing literature about Cminimal Legendrian surfaces into $\mathbb{S}^{5}$ and H-minimal Lagrangian surfaces into $\mathbb{C P}^{2}$. In particular, the existence of many minimal and $\mathrm{H}$ minimal Lagrangian tori immersed in $\mathbb{C P}^{2}$ is proved respectively in [17] in [5], while a family of H-minimal Lagrangian tori in $\mathbb{C P}^{2}$ with $\mathbb{S}^{1}$-symmetry is explicitly described in [21] and [22]. However, as discussed in Section 2.1, the topological type of the Legendrian lifts are no longer the torus a priori, but rather its universal covering, i.e. the plane.

4.3.3. Case $n \geq 4$. Besides the trivial example of the totally geodesic Legendrian embedding of $\mathbb{S}^{n-1}$ into $\mathbb{S}^{2 n-1}$, we point out that compact minimal Legendrian immersions of $\mathbb{S}^{1} \times \mathbb{S}^{n-2}$ into $\mathbb{S}^{2 n-1}$ have been constructed in [2]. On the other hand a construction of C-minimal Legendrian immersions (including non-minimal ones) has been described in Section 3 of [8] as follows: let $n_{1}, n_{2}$ be two integer numbers such that $n_{1}+n_{2}=n, \gamma=\left(\gamma_{1}, \gamma_{2}\right): I \rightarrow \mathbb{S}^{3}$ a Legendrian curve which is solution of one of the one-parametre family of o.d.e.

$$
\left(\gamma_{j}^{\prime} \overline{\gamma_{j}}\right)(t)=(-1)^{j-1} i e^{i \mu t} \overline{\gamma_{1}}(t)^{n_{1}} \overline{\gamma_{2}}(t)^{n_{2}}, \mu \in \mathbb{R}, j=1,2,
$$


and $\psi_{1}$ and $\psi_{2}$ two C-minimal Legendrian immersions of orientable manifolds $N_{1}$ and $N_{2}$ of dimensions $n_{1}-1$ and $n_{2}-1$ into $\mathbb{S}^{2 n_{1}-1}$ and $\mathbb{S}^{2 n_{2}-1}$ respectively. Then the following immersion

$$
\begin{array}{rlc}
\Psi: I \times N_{1} \times N_{2} & \longrightarrow & \mathbb{S}^{2 n-1}, \\
(t, x, y) & \mapsto \quad\left(\gamma_{1}(t) \psi_{1}(x), \gamma_{2}(t) \psi_{2}(y)\right),
\end{array}
$$

is $\mathrm{C}$-minimal.

While it turns out to be difficult to describe the general solution of (14), and therefore to control its closedness, we point out that the Legendrian curve with constant curvature $c=\sqrt{n_{2} / n_{1}}-\sqrt{n_{1} / n_{2}}$ (cf Section 4.3.1)

$$
\gamma_{n_{1}, n_{2}}(s)=\frac{1}{\sqrt{n_{1}+n_{2}}}\left(\sqrt{n_{1}} e^{i \sqrt{\frac{n_{2}}{n_{1}}} s}, \sqrt{n_{2}} e^{-i \sqrt{\frac{n_{1}}{n_{2}}} s}\right)
$$

is a closed solution of Equation (14) with parametre $\mu=0$.

Hence, given two compact C-minimal Legendrian immersions of two manifolds $N_{1}$ and $N_{2}$ into $\mathbb{S}^{2 n_{1}-1}$ and $\mathbb{S}^{2 n_{2}-1}$ respectively, we are able to construct a compact immersion of C-minimal immersion of $\mathbb{S}^{1} \times N_{1} \times N_{2}$ into $\mathbb{S}^{2 n-1}$, where $n=n_{1}+n_{2}$. We may start with simple examples such as totally geodesic embedding, and iterate the process in order to get immersions of a number of topological types:

Corollary 3. Given $n$ integer numbers $m_{1}, \ldots, m_{n}$, there exist $C$-minimal immersions of $\mathbb{T}^{n} \times \prod_{j=1}^{n} \mathbb{S}^{m_{j}}$ into $\mathbb{S}^{2 N-1}$, where $N=n+\sum_{j=1}^{n} m_{j}$.

\section{Construction with a Lagrangian surface And TWO LEGENDRIAN IMMERSIONS}

5.1. The construction. In this section we give a detailed study of the geometry of a family of Lagrangian submanifolds introduced in [11].

Theorem 2. Let $\phi=\left(\phi_{1}, \phi_{2}\right)$ be a Lagrangian immersion of an orientable surface $\Sigma$ into $\mathbb{C}^{2}$ such that $\phi_{1}$ and $\phi_{2}$ do not vanish, and $\psi_{1}, \psi_{2}$ two Legendrian immersions of orientable manifolds $N_{1}$ and $N_{2}$ into $\mathbb{S}^{2 n_{1}-1}$ and $\mathbb{S}^{2 n_{2}-1}$, with $n_{1}+n_{2}=n$. Then the map

$$
\begin{aligned}
& \Phi: \Sigma \times N_{1} \times N_{2} \longrightarrow \quad \mathbb{C}^{n} \\
& (p, x, y) \quad \longmapsto \quad\left(\phi_{1}(p) \psi_{1}(x), \phi_{2}(p) \psi_{2}(y)\right)
\end{aligned}
$$

is a Lagrangian immersion with induced metric

$$
\bar{g}=g+\left|\phi_{1}\right|^{2} g_{1}+\left|\phi_{2}\right|^{2} g_{2},
$$


where $g, g_{1}$ and $g_{2}$ are the induced metric on $\Sigma, N_{1}$ and $N_{2}$ respectively, and whose Lagrangian angle map is

$$
\beta_{\Phi}(p, x, y)=\left(n_{1}-1\right) \pi+G_{\phi}(p)+\beta_{\psi_{1}}(x)+\beta_{\psi_{2}}(y),
$$

where $\beta_{\psi_{1}}$ and $\beta_{\psi_{2}}$ are the Legendrian angle maps of $\psi_{1}$ and $\psi_{2}$ and $G_{\phi}$ is the $\mathbb{R} / 2 \pi \mathbb{Z}$-valued map defined on $\Sigma$ by

$$
G_{\phi}:=\beta_{\phi}+\left(n_{1}-1\right) \arg \phi_{1}+\left(n_{2}-1\right) \arg \phi_{2} .
$$

In addition the gradient of $\beta_{\Phi}$ is given by

$$
\nabla_{\bar{g}} \beta_{\Phi}=\left(\nabla_{g} G_{\phi},\left|\phi_{1}\right|^{-2} \nabla_{g_{1}} \beta_{\psi_{1}},\left|\phi_{2}\right|^{-2} \nabla_{g_{2}} \beta_{\psi_{2}}\right) .
$$

Moreover, given $X, Y$ and $Z$ three vector fields on $\Sigma, N_{1}$ and $N_{2}$ respectively, and denoting by $\bar{D}$ (resp. by $D, D^{1}, D^{2}$ ) the Levi-Civita connection of $\bar{g}$ (resp. of $\left.g, g_{1}, g_{2}\right)$, we have

$$
\bar{D}_{(X, 0,0)} \nabla_{\bar{g}} \beta_{\Phi}=\left(D_{X} \nabla_{g} G_{\phi}, X\left(\left|\phi_{1}\right|^{-2}\right) \nabla_{g_{1}} \beta_{\psi_{1}}, X\left(\left|\phi_{2}\right|^{-2}\right) \nabla_{g_{2}} \beta_{\psi_{2}}\right),
$$

$\bar{D}_{(0, Y, 0)} \nabla_{\bar{g}} \beta_{\Phi}=\left(\frac{\nabla_{g}\left|\phi_{1}\right|^{-2}}{\left|\phi_{1}\right|^{2}} d \beta_{\psi_{1}}(Y), \frac{D_{Y}^{1} \nabla_{g_{1}} \beta_{\psi_{1}}}{\left|\phi_{1}\right|^{2}}+g\left(\nabla_{g} G_{\phi}, \nabla_{g}\left|\phi_{1}\right|^{-2}\right) Y, 0\right)$,

$\bar{D}_{(0,0, Z)} \nabla_{\bar{g}} \beta_{\Phi}=\left(\frac{\nabla_{g}\left|\phi_{2}\right|^{-2}}{\left|\phi_{2}\right|^{2}} d \beta_{\psi_{2}}(Z), 0, \frac{D_{Z}^{2} \nabla_{g_{2}} \beta_{\psi_{2}}}{\left|\phi_{2}\right|^{2}}+g\left(\nabla_{g} G_{\phi}, \nabla_{g}\left|\phi_{2}\right|^{-2}\right) Z\right)$ and

$\Delta_{\bar{g}} \beta_{\Phi}=\frac{1}{\left|\phi_{1}\right|^{n_{1}-1}\left|\phi_{2}\right|^{n_{2}-1}} \operatorname{div}_{g}\left(\left|\phi_{1}\right|^{n_{1}-1}\left|\phi_{2}\right|^{n_{2}-1} \nabla_{g} G_{\phi}\right)+\frac{\Delta_{g_{1}} \psi_{1}}{\left|\phi_{1}\right|^{2}}+\frac{\Delta_{g_{2}} \psi_{2}}{\left|\phi_{2}\right|^{2}}$.

Proof. For the computation of the Lagrangian angle of the immersion $\Phi$, we refer to [11].

Let $\left(t_{\mu}\right), \mu=1,2,\left(x_{a}\right), 1 \leq a \leq n_{1}-1$ and $\left(y_{j}\right), 1 \leq j \leq n_{2}-1$ be local coordinates on $\Sigma, N_{1}$ and $N_{2}$ respectively. We shall denote by $\left(z_{\alpha}\right)=\left(t_{\mu}, x_{a}, y_{j}\right)$ the resulting coordinates on $\Sigma \times N_{1} \times N_{2}$. It is straightforward that

$$
\bar{g}=g+\left|\phi_{1}\right|^{2} g_{1}+\left|\phi_{2}\right|^{2} g_{2} .
$$

Thus, using that the matrix of $\bar{g}$ in the coordinates $\left(z_{\alpha}\right)$ is blockdiagonal, we compute:

$$
\nabla_{\bar{g}} \beta_{\Phi}=\sum_{\alpha, \beta=1}^{n} \bar{g}^{\alpha \beta} \frac{\partial \beta_{\Phi}}{\partial z_{\beta}} \partial z_{\alpha}
$$




$$
\begin{gathered}
=\sum_{\mu, \nu=1}^{2} g^{\mu \nu} \frac{\partial G_{\phi}}{\partial t_{\mu}}\left(\partial_{t_{\nu}}, 0,0\right)+\left|\phi_{1}\right|^{-2} \sum_{a, b=1}^{n_{1}-1} g^{a b} \frac{\partial \beta_{\psi_{1}}}{\partial x_{b}}\left(0, \partial_{x_{a}}, 0\right) \\
+\left|\phi_{2}\right|^{-2} \sum_{j, k=1}^{n_{2}-1} g^{j k} \frac{\partial \beta_{\psi_{2}}}{\partial y_{k}}\left(0,0, \partial_{y_{j}}\right), \\
=\left(\nabla_{g} G_{\phi}, 0,0\right)+\left|\phi_{1}\right|^{-2}\left(0, \nabla_{g_{1}} \beta_{\psi_{1}}, 0\right)+\left|\phi_{2}\right|^{-2}\left(0,0, \nabla_{g_{2}} \beta_{\psi_{2}}\right) .
\end{gathered}
$$

Next, a long but straightforward computation gives

$$
\begin{aligned}
& \bar{D}_{\partial_{t_{\mu}}} \partial_{t_{\nu}}=D_{\partial_{t_{\mu}}} \partial_{t_{\nu}}, \quad \bar{D}_{\partial_{x_{a}}} \partial_{t_{\mu}}=\bar{D}_{\partial_{t_{\mu}}} \partial_{x_{a}}=\frac{\partial\left(\left|\phi_{1}\right|^{-2}\right)}{\partial t_{\mu}} \partial_{x_{a}}, \\
& \bar{D}_{\partial_{y_{j}}} \partial_{t_{\mu}}=\bar{D}_{\partial_{t_{\mu}}} \partial_{y_{j}}=\frac{\partial\left(\left|\phi_{2}\right|^{-2}\right)}{\partial t_{\mu}} \partial_{y_{j}} \\
& \bar{D}_{\partial_{x_{a}}} \partial_{x_{b}}=\nabla_{g}\left(\left|\phi_{1}\right|^{-2}\right) g_{a b}+D_{\partial_{x_{a}}}^{1} \partial_{x_{b}} \text {, } \\
& \bar{D}_{\partial_{y_{j}}} \partial_{y_{k}}=\nabla_{g}\left(\left|\phi_{2}\right|^{-2}\right) g_{j k}+D_{\partial_{y_{j}}}^{2} \partial_{y_{k}} \text {. }
\end{aligned}
$$

From these equations it not difficult to obtain Equations (17), (18) and (19). It remains to compute the Laplacian of $\beta_{\Phi}$ :

$$
\begin{gathered}
\Delta_{\bar{g}} \beta_{\Phi}=\sum_{\alpha, \beta=1}^{n} \bar{g}\left(\bar{D}_{\partial_{z_{\alpha}}} \nabla_{\bar{g}} \beta_{\Phi}, \partial_{z_{\beta}}\right) \bar{g}^{\alpha \beta} \\
=\sum_{\mu, \nu=1}^{2} \bar{g}\left(\bar{D}_{\partial_{t_{\mu}}} \nabla_{\bar{g}} \beta_{\Phi}, \partial_{t_{\nu}}\right) \bar{g}^{\mu \nu}+\sum_{a, b=1}^{n_{1}-1} \bar{g}\left(\bar{D}_{\partial_{x_{a}}} \nabla_{\bar{g}} \beta_{\Phi}, \partial_{x_{b}}\right) \bar{g}^{a b}+\sum_{j, k=1}^{n_{2}-1} \bar{g}\left(\bar{D}_{\partial_{y_{j}}} \nabla_{\bar{g}} \beta_{\Phi}, \partial_{y_{k}}\right) \bar{g}^{j k} \\
=\sum_{\mu, \nu=1}^{2} g\left(\bar{D}_{\partial_{t_{\mu}}} \nabla_{\bar{g}} G_{\phi}, \partial_{t_{\nu}}\right) g^{\mu \nu}+\sum_{a, b=1}^{n_{1}-1} g_{1}\left(\bar{D}_{\partial_{x_{a}}} \nabla_{\bar{g}} \beta_{\Phi}, \partial_{x_{b}}\right) g^{a b}+\sum_{j, k=1}^{n_{2}-1} g_{2}\left(\bar{D}_{\partial_{y_{j}}} \nabla_{\bar{g}} \beta_{\Phi}, \partial_{y_{k}}\right) g^{j k} \\
=\Delta_{g} G_{\phi}+\sum_{a, b=1}^{n_{1}-1}\left(\frac{1}{\left|\phi_{1}\right|^{2}} g_{1}\left(D_{\partial_{x_{a}}}^{1} \nabla_{g_{1}} \beta_{\psi_{1}}, \partial_{x_{b}}\right)+g\left(\nabla_{g} G_{\phi}, \nabla_{g}\left|\phi_{1}\right|^{-2}\right) g_{a b}\right) g^{a b} \\
+\sum_{j, k=1}^{n_{2}-1}\left(\frac{1}{\left|\phi_{2}\right|^{2}} g_{2}\left(D_{\partial_{y_{j}}}^{2} \nabla_{g_{2}} \beta_{\psi_{2}}, \partial_{y_{k}}\right)+g\left(\nabla_{g} G_{\phi}, \nabla_{g}\left|\phi_{2}\right|^{-2}\right) g_{j k}\right) g^{j k} \\
=\Delta_{g} G_{\phi}+\frac{1}{\left|\phi_{1}\right|^{2}} \Delta_{g_{1}} \beta_{\psi_{1}}+\left(n_{1}-1\right) g\left(\nabla_{g} G_{\phi}, \nabla_{g}\left|\phi_{1}\right|^{-2}\right) \\
+\frac{1}{\left|\phi_{2}\right|^{2}} \Delta_{g_{2}} \beta_{\psi_{2}}+\left(n_{2}-1\right) g\left(\nabla_{g} G_{\phi}, \nabla_{g}\left|\phi_{2}\right|^{-2}\right) .
\end{gathered}
$$

Finally, we check that

$$
\frac{1}{\left|\phi_{1}\right|^{n_{1}-1}\left|\phi_{2}\right|^{n_{2}-1}} \operatorname{div}_{g}\left(\left|\phi_{1}\right|^{n_{1}-1}\left|\phi_{2}\right|^{n_{2}-1} \nabla_{g} G_{\phi}\right)
$$




$$
=\Delta_{g} G_{\phi}+\left(n_{1}-1\right) g\left(\nabla_{g} G_{\phi}, \nabla_{g}\left|\phi_{1}\right|^{-2}\right)+\left(n_{2}-1\right) g\left(\nabla_{g} G_{\phi}, \nabla_{g}\left|\phi_{2}\right|^{-2}\right),
$$

which completes the proof.

Remark 3. If the Lagrangian immersion $\phi$ is the product of two planar curves $\left(\alpha_{1}, \alpha_{2}\right)$, then $\Phi$ is the product of two Lagrangian immersions $\Phi_{1}$ and $\Phi_{2}$ of the type described in Theorem 1. This fact will be important for the classification of immersions $\Phi$ with parallel mean curvature vector (Corollary 4).

If the Lagrangian immersion is of the type of Theorem 1, i.e. $\phi=\alpha \gamma$ where $\alpha$ is a planar curve and $\gamma$ is a Legendrian curve in $\mathbb{S}^{3}$, then the immersion $\Phi$ is also of the type of Theorem 1 , with $\psi=\left(\gamma_{1} \psi_{1}, \gamma_{2} \psi_{2}\right)$ is of the type described in [8] (see also Section 4.3.3). Moreover, any Lagrangian immersion in $\mathbb{C}^{n}$ invariant under the action of $S O\left(n_{1}\right) \times$ $S O\left(n_{2}\right)$, with $n_{1}+n_{2}=n$ and $n_{1}, n_{2} \geq 2$, is congruent to an open subset of one of the Lagrangian submanifolds of Theorem 2, where $\psi_{1}(x)=x$ and $\psi_{2}(y)=y$ are the totally geodesic embeddings of $\mathbb{S}^{n_{i}-1}$ into $\mathbb{S}^{2 n_{i}-1}, i=1,2$.

The Lagrangian immersions described in Theorem 2 have singularities at the points $(p, x, y) \in \Sigma \times N_{1} \times N_{2}$ where either $\phi_{1}(p)=0$ or $\phi_{2}(p)=0$.

It has been proved in [11] (and it follows from Equation (15)) that the immersion $\Phi$ described in Theorem 2 is minimal if and only if $\psi_{1}$ are $\psi_{2}$ are minimal and $G_{\phi}$ is constant. If $n=3$, then either $\left(n_{1}, n_{2}\right)=(2,1)$ or $(1,2)$. In both cases, $\psi_{1}$ or $\psi_{2}$ must be a Legendrian geodesic in $\mathbb{S}^{3}$, which can be parameterized, up to congruence by $\psi(t)=\frac{1}{\sqrt{2}}\left(e^{i t}, e^{-i t}\right)$. The corresponding Lagrangian immersion $\Phi$ takes the following form:

$$
\begin{gathered}
\Phi: \Sigma \times \mathbb{R} \longrightarrow \mathbb{C}^{3} \\
(p, t) \mapsto\left(\frac{\phi_{1}(p)}{\sqrt{2}} e^{i t}, \frac{\phi_{1}(p)}{\sqrt{2}} e^{-i t}, \phi_{2}(p)\right) .
\end{gathered}
$$

These examples, which are invariant under the action of $U(1) \equiv S O(2)$ on $\mathbb{C}^{3}$ given by

$$
e^{i t} \cdot\left(z_{1}, z_{2}, z_{3}\right)=\left(e^{i t} z_{1}, e^{-i t} z_{2}, z_{3}\right)
$$

have been studied in detail by Joyce in [19] in the minimal Lagrangian case.

Before we characterize those immersions $\Phi$ which have parallel mean curvature vector or are compact and H-minimal, we need a technical lemma:

Lemma 1. Let $\Sigma$ be a connected, properly immersed Lagrangian surface of $\mathbb{C}^{2}$ with coordinates $\left(\phi_{1}, \phi_{2}\right)$ and such that the vector fields $\nabla_{g}\left|\phi_{1}\right|$ 
and $\nabla_{g}\left|\phi_{2}\right|$ are linearly dependent. Then either $\Sigma$ is contained in a product of two planar curves $\alpha_{1}$ and $\alpha_{2}$ (one of them being an arc of circle), or it may be locally parametrized by an immersion of the form

$$
\phi(s, t)=\left(\sqrt{a x(s)+b} e^{i(s+t)}, \sqrt{x(s)} e^{i(s-a t)}\right) .
$$

where $x(s)>0$ and $(a, b) \in \mathbb{Q} \times \mathbb{R}$.

Proof. Suppose first that $\left|\phi_{1}\right|$ is constant. We proceed by contradiction assuming that $\Sigma$ is not a product of curves. Then it may be locally parametrized by

$$
\phi(s, t)=\left(C e^{i \theta(s, t)}, s+i t\right)
$$

where $s+i t$ belongs to an open subset of $\mathbb{C}, C$ is a positive constant and $\theta$ an $\mathbb{R} / 2 \pi \mathbb{Z}$-valued map. If follows that $\omega\left(\phi_{s}, \phi_{t}\right)=1$, which contradicts the Lagrangian assumption. Of course the argument is the same if $\left|\phi_{2}\right|$ is constant.

It remains to treat the case in which $\left|\phi_{1}\right|$ and $\left|\phi_{2}\right|$ are not constant. There exists therefore a smooth real map $u$ such that $\left|\phi_{1}\right|^{2}=u\left(\left|\phi_{2}\right|^{2}\right)$. Introduce the hypersurface

$$
Q:=\left\{\left(z_{1}, z_{2}\right) \in \mathbb{C}^{2},\left|z_{1}\right|^{2}-u\left(\left|z_{2}\right|^{2}\right)=0\right\} .
$$

Since $\Sigma \subset Q$, we have $T^{\perp} Q \subset T^{\perp} \Sigma$. On the other hand, at a point $\left(z_{1}, z_{2}\right)$ of $Q, T_{\left(z_{1}, z_{2}\right)}^{\perp} Q=\left(z_{1},-u^{\prime}\left(\left|z_{2}\right|^{2}\right) z_{2}\right) \mathbb{R}$, thus $\left(z_{1},-u^{\prime}\left(\left|z_{2}\right|^{2}\right) z_{2}\right) \in$ $T_{\left(z_{1}, z_{2}\right)}^{\perp} \Sigma$. By the Lagrangian assumption, the vector field $V\left(z_{1}, z_{2}\right)=$ $\left(i z_{1},-i u^{\prime}\left(\left|z_{2}\right|^{2}\right) z_{2}\right)$ is tangent to $\Sigma$, so its integral curves

$$
t \mapsto\left(z_{1}^{0} e^{i t}, z_{2}^{0} e^{-i u^{\prime}\left(\left|z_{2}^{0}\right|^{2}\right) t}\right),
$$

with initial point $\left(z_{1}^{0}, z_{2}^{0}\right) \in \Sigma$, are contained in $\Sigma$. It follows that $\Sigma$ may be locally parametrized by

$$
\phi(s, t)=\left(z_{1}(s) e^{i t}, z_{2}(s) e^{-i u^{\prime}\left(\left|z_{2}(s)\right|^{2}\right) t}\right),
$$

where $\left(z_{1}(s), z_{2}(s)\right)$ is a curve in $\Sigma$ tranversal to the integral curves of the vector field $V$. Moreover, we may replace the curve $\left(z_{1}(s), z_{2}(s)\right)$ by $\left(\tilde{z}_{1}(s), \tilde{z}_{2}(s)\right)=\left(z_{1}(s) e^{i \theta(s)}, z_{2}(s) e^{-i u^{\prime}\left(\left|z_{2}(s)\right|^{2}\right) \theta(s)}\right)$ without changing the image of $\phi$. A routine computation shows that we can choose the function $\theta(s)$ in order to have $\arg \tilde{z}_{1}=\arg \tilde{z}_{2}$. Using the assumption $\left(\tilde{z}_{1}(s), \tilde{z}_{2}(s)\right) \in Q$, we deduce that $\tilde{z}_{1}(s)=\sqrt{u\left(\left|\tilde{z}_{2}(s)\right|^{2}\right)} \tilde{z}_{2}(s)$ so, writing $\tilde{z}_{2}(s)=\sqrt{x(s)} e^{i s}$, we have $\tilde{z}_{1}(s)=\sqrt{u(x(s))} e^{i s}$ and we obtain

$$
\phi(s, t)=\left(\sqrt{u(x(s))} e^{i(s+t)}, \sqrt{x(s)} e^{i\left(s-u^{\prime}(x(s)) t\right)} .\right.
$$

We claim now that the properly immersed assumption imposes a restriction on $u$ : if $u^{\prime}\left(\left|z_{2}^{0}\right|^{2}\right)$ does not belong to $\mathbb{Q}$, then the integral curves of $V$ are not closed; more precisely they are dense in the torus 
$\left\{\left(z_{1}, z_{2}\right) \in \mathbb{C}^{2}|| z_{1}|=| z_{1}^{0}|,| z_{2}|=| z_{2}^{0} \mid\right\}$. Since $\Sigma$ is assumed to be properly immersed, it must be contained in this torus, which is a product of curves. If it is not the case, $u^{\prime}\left(\left|z_{2}^{0}\right|^{2}\right)$ must be rationaly related to $\mathbb{Q}$, so it is constant and $u(x)=a x+b$, where $(a, b) \in \mathbb{Q} \times \mathbb{R}$, which implies the claimed formula.

Corollary 4. Consider the Lagrangian immersion

$$
\begin{aligned}
& \Phi: \Sigma \times N_{1} \times N_{2} \longrightarrow \quad \mathbb{C}^{n} \\
& (p, x, y) \quad \longmapsto \quad\left(\phi_{1}(p) \psi_{1}(x), \phi_{2}(p) \psi_{2}(y)\right)
\end{aligned}
$$

where $\phi=\left(\phi_{1}, \phi_{2}\right): \Sigma \rightarrow \mathbb{C}^{2}$ is a Lagrangian immersion of an orientable, properly immersed surface $\Sigma$ with Lagrangian angle $\beta_{\phi}$ and $\psi_{i}$, $i=1,2$ are two Legendrian immersions of orientable manifolds $N_{i}$ into $\mathbb{S}^{2 n_{i}-1}$, where $n_{1}+n_{2}=n$. Then $\Phi$ has parallel mean curvature vector if and only if

- either (i) it is minimal; in this case the immersion $\phi$ is such that $G_{\phi}:=\beta_{\phi}+\left(n_{1}-1\right) \arg \phi_{1}+\left(n_{2}-1\right) \arg \phi_{2}$ is constant and the immersions $\psi_{1}$ and $\psi_{2}$ are minimal (see [11]);

- or (ii) it is a product $\Phi=\left(\Phi_{1}, \Phi_{2}\right)$ of two Lagrangian immersions into $\mathbb{C}^{n_{1}}$ and $\mathbb{C}^{n_{2}}$ with parallel mean curvature vector (as described in Theorem 1); in this case the immersion $\phi$ is a product of two planar curves $\left(\alpha_{1}, \alpha_{2}\right)$;

- or (iii) $\phi$ is, up to scaling, the immersion

$$
\phi(s, t)=\left(\cos s e^{i t}, \sin s e^{i t}\right)
$$

and the immersions $\psi_{1}$ and $\psi_{2}$ are minimal.

Moreover, if $N_{1}$ and $N_{2}$ are compact, the immersion $\Phi$ is H-minimal if and only if the immersion $\phi$ is such that the vector field

$$
A_{\phi}:=\left|\phi_{1}\right|^{n_{1}-1}\left|\phi_{2}\right|^{n_{2}-1} \nabla_{g} G_{\phi}
$$

is divergence free and the immersions $\psi_{1}$ and $\psi_{2}$ are $C$-minimal.

Proof. Assume that $\left|\phi_{1}\right|$ and $\left|\phi_{2}\right|$ are not constant. The fact that $\nabla_{\bar{g}} \beta_{\Phi}$ is parallel and Equations (17), (18) and (19) of Theorem 2 imply that $\beta_{\psi_{1}}$ and $\beta_{\psi_{2}}$ are constant and that

$$
\begin{aligned}
& g\left(\nabla_{g} G_{\phi}, \nabla_{g}\left|\phi_{1}\right|^{-2}\right)=0, \\
& g\left(\nabla_{g} G_{\phi}, \nabla_{g}\left|\phi_{2}\right|^{-2}\right)=0 .
\end{aligned}
$$

Hence, if the vector fields $\nabla_{g}\left|\phi_{1}\right|^{-2}$ and $\nabla_{g}\left|\phi_{2}\right|^{-2}$ are independent, the vector field $\nabla_{g} G_{\phi}$ vanishes, so the map $G_{\phi}$ is constant. By Equation (15) of Theorem 2, it follows that the immersion $\Phi$ is minimal and we are in case $(i)$. We now assume that $G_{\phi}$ is not constant and that 
the vector fields $\nabla_{g}\left|\phi_{1}\right|^{-2}$ and $\nabla_{g}\left|\phi_{2}\right|^{-2}$ are linearly dependent. Using Lemma 1, and since $\left|\phi_{1}\right|$ and $\left|\phi_{2}\right|$ are not constant, the immersion $\phi$ takes the form

$$
\phi(s, t)=\left(\sqrt{a x(s)+b} e^{i(s+t)}, \sqrt{x(s)} e^{i(s-a t)}\right),
$$

where $x(s)>0$ is not constant and $(a, b) \in \mathbb{Q} \times \mathbb{R}$. We easily compute that

$$
G_{\phi}=\left(n_{1}+n_{2}\right) s+\left(n_{1}-a n_{2}\right) t-\arctan \left(\frac{x^{\prime}((1+a) a x+b)}{2(1+a) x(a x+b)}\right)
$$

and use the assumption that $\nabla^{g} G_{\phi}$ is parallel (coming from Equation (17)), more precisely, the vanishing of $g\left(\nabla_{\partial_{s}}^{g} G_{\phi}, \partial_{t}\right)$ and $g\left(\nabla_{\partial_{t}}^{g} G_{\phi}, \partial_{t}\right)$. A tedious but straightforward computation involving the Christoffel symbols of the induced metric $g$ shows that either $a=-1$ or $b$ vanishes. If $b$ vanishes the immersion $\phi$ takes the simpler form

$$
\phi(s, t)=\left(\sqrt{a} \alpha(s) e^{i t}, \alpha(s) e^{-i a t}\right),
$$

where $\alpha:=\sqrt{x(s)} e^{i s}$. Hence the immersion $\Phi$ can be written as $\Phi(s, t, x, y)=$ $\sqrt{1+a} \alpha(s) \psi(t, x, y)$, where

$$
\psi(t, x, y):=\frac{1}{\sqrt{1+a}}\left(\sqrt{a} e^{i t} \psi_{1}(x), e^{-i a t} \psi_{2}(y)\right)
$$

is a Legendrian immersion into $\mathbb{S}^{2 n-1}$. In other words, we are in the case of Section 4, and we can rely on the Corollary 2 to deduce that either the immersion $\Phi$ is minimal, which is case (i), unless the curve $\alpha$ is a circle. However this last case is excluded since $x$ is assumed to be not constant. If $a=-1$, setting $\sigma:=\arcsin \sqrt{x / b}$ and $\tau:=s+t$, the immersion $\phi$ becomes

$$
\phi(\sigma, \tau)=\left(b \cos \sigma e^{i \tau}, b \sin \sigma e^{i \tau}\right),
$$

so we are in case (iii). Finally, if one of the quantities $\left|\phi_{1}\right|$ and $\left|\phi_{2}\right|$ is constant, by the second point of Lemma 1, the immersion $\phi$ is a product of planar curves and we get the case (ii).

To complete the proof, we observe that if $\Phi$ is H-minimal, by Equation $(20), \Delta_{g_{1}} \beta_{\psi_{1}}$ and $\Delta_{g_{2}} \beta_{\psi_{2}}$ must be constant. By the compactness assumption, those constants must be zero, so the immersions $\psi_{1}$ and $\psi_{2}$ are $\mathrm{C}$-minimal and the immersion $\phi$ is such that the vector field $\left|\phi_{1}\right|^{n_{1}-1}\left|\phi_{2}\right|^{n_{2}-1} \nabla_{g} G_{\phi}$ is divergence free.

In Section 4.3 we have given a description of several compact, Cminimal Legendrian immersions in odd-dimensional spheres. On the other hand, it turns out to be difficult to solve the equation $\operatorname{div} A_{\phi}=0$ in full generality. In the next section we make use of a construction 
introduced in [7] in order to get a countable family of solutions to this equation.

\subsection{A special class of Lagrangian surfaces of $\mathbb{C}^{2}$ satisfying} $\operatorname{div} A_{\phi}=0$. We follow the notation of Section 2.3 and consider a Legendrian curve $\gamma: I_{1} \rightarrow \mathbb{S}^{3}$ and a Legendrian curve $\alpha: I_{2} \rightarrow \mathbb{H}_{1}^{3}$, that we both assume to be parametrized by arclength. Consider the map: $\phi: I_{1} \times I_{2} \subset \mathbb{R}^{2} \rightarrow \mathbb{C}^{2}=\mathbb{C} \times \mathbb{C}$ defined by

$$
\phi(s, t) \equiv \gamma(s) \odot \alpha(t)=\left(\gamma_{1}(s) \alpha_{1}(t), \gamma_{2}(s) \alpha_{2}(t)\right) .
$$

Then $\phi=\gamma \odot \alpha$ is a Lagrangian conformal immersion in $\mathbb{C}^{2}$ whose induced metric is given by

$$
g=\left(\left|\gamma_{1}\right|^{2}+\left|\alpha_{1}\right|^{2}\right)\left(d s^{2}+d t^{2}\right)=\left(\xi_{3}+\eta_{3}\right)\left(d s^{2}+d t^{2}\right) .
$$

The Lagrangian angle map $\beta_{\phi}$ of the Lagrangian conformal immersion $\phi=\gamma \odot \alpha$ and the Legendrian angles $\beta_{\gamma}$ and $\beta_{\alpha}$ of $\gamma$ and $\alpha$ are related by

$$
\beta_{\phi}(s, t)=\beta_{\gamma}(s)+\beta_{\alpha}(t)+\pi .
$$

Setting $G \equiv G_{\phi}$ for sake of brevity, we have

$G=\beta_{\gamma}+\left(n_{1}-1\right) \arg \gamma_{1}+\left(n_{2}-1\right) \arg \gamma_{2}+\beta_{\alpha}+\left(n_{1}-1\right) \arg \alpha_{1}+\left(n_{2}-1\right) \arg \alpha_{2}+\pi$.

On the other hand,

$$
A_{\phi}=\frac{\left(\left|\gamma_{1}\right|\left|\alpha_{1}\right|\right)^{n_{1}-1}\left(\left|\gamma_{2}\right|\left|\alpha_{2}\right|\right)^{n_{2}-1}}{\left|\gamma_{1}\right|^{2}+\left|\alpha_{1}\right|^{2}}\left(G_{s}, G_{t}\right)
$$

Hence we deduce that

$$
\begin{gathered}
\left(\left|\gamma_{1}\right|^{2}+\left|\alpha_{1}\right|^{2}\right) \operatorname{div} A_{\phi} \\
=\left(\left(\left|\gamma_{1}\right|\left|\alpha_{1}\right|\right)^{n_{1}-1}\left(\left|\gamma_{2}\right|\left|\alpha_{2}\right|\right)^{n_{2}-1} G_{s}\right)_{s}+\left(\left(\left|\gamma_{1}\right|\left|\alpha_{1}\right|\right)^{n_{1}-1}\left(\left|\gamma_{2}\right|\left|\alpha_{2}\right|\right)^{n_{2}-1} G_{t}\right)_{t} .
\end{gathered}
$$

A particular solution arises when the two terms of the right hand side vanish, i.e. if there exist two real constants $c_{1}$ and $c_{2}$ such that

$$
\begin{aligned}
& \left|\gamma_{1}\right|^{n_{1}-1}\left|\gamma_{2}\right|^{n_{2}-1} G_{s}=c_{1}, \\
& \left|\alpha_{1}\right|^{n_{1}-1}\left|\alpha_{2}\right|^{n_{2}-1} G_{t}=c_{2} .
\end{aligned}
$$

Since we have

$$
\begin{aligned}
& G_{s}=\beta_{\gamma}^{\prime}+\left\langle\gamma_{1}^{\prime}, J \gamma_{1}\right\rangle\left(\frac{n_{1}-1}{\left|\gamma_{1}\right|^{2}}-\frac{n_{2}-1}{\left|\gamma_{2}\right|^{2}}\right), \\
& G_{t}=\beta_{\alpha}^{\prime}+\left\langle\alpha_{1}^{\prime}, J \alpha_{1}\right\rangle\left(\frac{n_{1}-1}{\left|\alpha_{1}\right|^{2}}-\frac{n_{2}-1}{\left|\alpha_{2}\right|^{2}}\right),
\end{aligned}
$$


we obtain the following sufficient conditions in order $\phi=\gamma \odot \alpha$ to satisfy $\operatorname{div} A_{\phi}=0$ :

$$
\begin{aligned}
& \beta_{\gamma}^{\prime}+\left\langle\gamma_{1}^{\prime}, J \gamma_{1}\right\rangle\left(\frac{n_{1}-1}{\left|\gamma_{1}\right|^{2}}-\frac{n_{2}-1}{\left|\gamma_{2}\right|^{2}}\right)=\frac{c_{1}}{\left|\gamma_{1}\right|^{n_{1}-1}\left|\gamma_{2}\right|^{n_{2}-1}}, \\
& \beta_{\alpha}^{\prime}+\left\langle\alpha_{1}^{\prime}, J \alpha_{1}\right\rangle\left(\frac{n_{1}-1}{\left|\alpha_{1}\right|^{2}}-\frac{n_{2}-1}{\left|\alpha_{2}\right|^{2}}\right)=\frac{c_{2}}{\left|\alpha_{1}\right|^{n_{1}-1}\left|\alpha_{2}\right|^{n_{2}-1}} .
\end{aligned}
$$

Finally, using the relations between the Legendrian curves $\gamma$ and $\alpha$ and their Hopf projections $\xi=\Pi \circ \gamma$ and $\eta=\Pi \circ \alpha$, (cf Section 2.3) we get:

$k_{\xi}+\left(\xi \times \xi^{\prime}\right)_{3}\left(\frac{n_{1}-1}{1 / 2+\xi_{3}}-\frac{n_{2}-1}{1 / 2-\xi_{3}}\right)=\frac{c_{1}}{\left(1 / 2+\xi_{3}\right)^{\left(n_{1}-1\right) / 2}\left(1 / 2-\xi_{3}\right)^{\left(n_{2}-1\right) / 2}}$,

$k_{\eta}+\left(\eta \times \eta^{\prime}\right)_{3}\left(\frac{n_{1}-1}{\eta_{3}-1 / 2}+\frac{n_{2}-1}{\eta_{3}+1 / 2}\right)=\frac{c_{2}}{\left(\eta_{3}-1 / 2\right)^{\left(n_{1}-1\right) / 2}\left(1 / 2+\eta_{3}\right)^{\left(n_{2}-1\right) / 2}}$.

The Legendrian lift $\gamma$ or $\alpha$ of a generic solution of Equation (30) or (31) is not expected to be closed, even if the projected curve $\xi$ or $\eta$ is so. Since we are interested in compact examples, we point out that the geodesic $\xi_{0}=\Pi \circ \gamma_{0}$, where $\gamma_{0}(s)=(\cos s, \sin s)$ trivially verifies $(28)$ with $c_{1}=0$ and the constant curvature curve $\eta_{\delta}(t)=\Pi\left(\alpha_{\delta}(t)\right)$, with

$$
\alpha_{\delta}(t)=\left(\sinh \delta e^{i \operatorname{coth} \delta t}, \cosh \delta e^{i \tanh \delta t}\right), \delta>0
$$

is a solution of (29) for a suitable constant $c_{2}(\delta)$ depending on $\delta$. Moreover $\alpha_{\delta}$ is a closed curve if and only if $\tanh ^{2} \delta=q / r \in \mathbb{Q}, 0<q<r$. In such a case $\alpha_{\delta}$ takes the form

$$
\alpha_{q, r}(t)=\frac{1}{\sqrt{r-q}}\left(\sqrt{q} e^{i \sqrt{r / q} t}, \sqrt{r} e^{i \sqrt{q / r} t}\right), 0<q<r .
$$

It follows that the corresponding immersions $\phi_{q, r}:=\gamma_{0} \odot \alpha_{q, r}$ are doubly periodic and their images are non-trivial Lagrangian tori. These tori, besides being solutions of $\operatorname{div} A_{\phi}=0$, turn out to be $\mathrm{H}$-minimal and self-similar for the mean curvature flow as well (cf [6]).

Summing up, using the two constructions of H-minimal Lagrangian immersions (Corollaries 2 and 4 ) and the existence result of C-minimal Legendrian immersions (Corollary 3), we are able to construct families of compact H-minimal Lagrangian immersions of various topological types. In the first case, the Lagrangian immersions $\Phi_{p}=\alpha_{p} \psi, p \in \mathbb{N}$, constructed using the countable family of closed curves $\alpha_{p}$ described 
in Section 4.2 and a $C$-minimal Legendrian immersion $\psi$ do not have parallel mean curvature vector by Corollary 2. Analogously, since the tori $\phi_{q, r}$ are not minimal and are not a Cartesian product of planar curves, the immersions $\Phi_{q, r}$ do not have parallel mean curvature by Corollary 4. Hence, we have got:

Corollary 5. Given $n$ integer numbers $m_{1}, \ldots, m_{n}$, there exist two countable families of compact immersions $\Phi_{p}$ and $\Phi_{q, r}$ of $\mathbb{T}^{n} \times \prod_{j=1}^{n} \mathbb{S}^{m_{j}}$ into $\mathbb{C}^{N}$, where $N=n+\sum_{j=1}^{n} m_{j}$, which are H-minimal and non trivial, i.e. non minimal and whose mean curvature vector is not parallel.

\section{REFERENCES}

[1] H. Anciaux, Construction of many Hamiltonian stationary Lagrangian surfaces in Euclidean four-space, Calc. of Var. 17 (2003), 105-120.

[2] H. Anciaux, Legendrian submanifolds foliated by $(n-1)$-spheres in $\mathbb{S}^{2 n+1}$, Matemática Contemporânea 30 (2006), 41-61.

[3] H. Anciaux \& P. Romon Cyclic and ruled Lagrangian surfaces in complex Euclidean space, Bull. of the Brazilian Math. Society, 40 (2009), no. 3, 341369

[4] H. Anciaux, I. Castro \& P. Romon, Lagrangian submanifolds foliated by $(n-1)$ spheres in $\mathbb{R}^{2 n}$, Acta Math. Sinica (English Series) 22 (2006), no 4, 1197-1214

[5] E. Carberry \& I. McIntosh, Special Lagrangian tori in $\mathbb{C P}^{2}$ come in real families of every dimension, J. London Math. Soc. (2) 69 no. 2, (2004), 531-544

[6] I. Castro \& A. Lerma, Hamiltonian stationary self-similar solutions for Lagrangian mean curvature flow in complex Euclidean plane, Proc. of the AMS, 138 (2010), 1821-1832

[7] I. Castro \& B.-Y. Chen, Lagrangian surfaces in complex Euclidean plane via spherical and hyperbolic curves, Tohoku Math. J. (2) 58 (2006), no. 4, 565-579

[8] I. Castro, H. Li \& F. Urbano, Hamiltonian-minimal Lagrangian submanifolds in complex space forms, Pacific J. Math. 227 (2006), no 1, 43-63

[9] I. Castro \& F. Urbano, Examples of unstable Hamiltonian-minimal Lagrangian tori in $\mathbb{C}^{2}$, Compositio Math. 111 (1998), 1-14

[10] I. Castro \& F. Urbano, On a minimal Lagrangian submanifold of $\mathbb{C}^{n}$ foliated by spheres, Michigan Math. J. 45 (1999), 71-82

[11] I. Castro \& F. Urbano, On a new construction of special Lagrangian immersions in complex Euclidean space, Quarter. J. Math. 55 (2004), 253-266

[12] R. Harvey \& H.B. Lawson, Calibrated geometries, Acta Math. 148 (1982), $47-157$

[13] M. Haskins, Special Lagrangian cones, Amer. J. Math. 126 (2004), 845-871

[14] M. Haskins, The geometric complexity of special Lagrangian $T^{2}$-cones, Invent. Math. 157 (2004), 11-70

[15] M. Haskins, N. Kapouleas, Gluing constructions of special Lagrangian cones. Handbook of geometric analysis. No. 1, 77-145, Adv. Lect. Math. (ALM), 7, Int. Press, Somerville, MA, 2008

[16] F. Hélein \& P. Romon, Weierstrass representation of Lagrangian surfaces in four-dimensional space using spinors and quaternions, Comm. Math. Helvetici 75 (2000), 668-680 
[17] F. Hélein \& P. Romon, Hamiltonian stationary tori in the complex projective plane, Proc. of the London Math. Soc. (2) 90 (2005) 472-496

[18] D.D. Joyce, Special Lagrangian $m$-folds in $\mathbb{C}^{m}$ with symmetries, Duke Math. J. 115 (2002), 1-51

[19] D.D. Joyce, U(1)-invariant special Lagrangian 3-folds. I. Nonsingular solutions, Adv. Math. 192 (2005), no 1, 35-71

[20] D. Joyce, Y.-I. Lee \& R. Schoen, On the existence of Hamiltonian stationary Lagrangian submanifolds in symplectic manifolds, arXiv:0902.3338

[21] H. Ma \& M. Schmies, Examples of Hamiltonian stationary Lagrangian submanifolds in $\mathbb{C} P^{2}$, Geom. Dedicata 118 (2006), 173-183

[22] A. E. Mironov, On the Hamiltonian-minimal Lagrangian tori in $\mathbb{C} P^{2}$, (translated from Russian) Siberian Math. J. 44 (2003), no 6, 1039-1042

[23] A. E. Mironov, On new examples of Hamiltonian-minimal and minimal Lagrangian submanifolds in $\mathbb{C}^{n}$ and $\mathbb{C} P^{n}$, (translated from Russian) Siberian Math. J. 195 (2004), 85-96

[24] Y.G. Oh, Second variation and stabilities of minimal Lagrangian submanifolds, Invent. Math. 101 (1990), 501-519

[25] U. Pinkall, Hopf tori in $\mathbb{S}^{3}$, Invent. Math. 81 (1985), no 2, 379-386

[26] A. Ros \& F. Urbano, Lagrangian submanifolds of $\mathbb{C}^{n}$ with conformal Maslov form and the Whitney sphere, J. Math. Soc. Japan 50 (1998), 203-226

[27] R. Schoen \& J. Wolfson, Minimizing volume among Lagrangian submanifolds, Proc. Symp. Pure Math. 65, Amer. Math. Soc., Providence, RI (1999)

[28] J. Wolfson, Minimal Lagrangian diffeomorphisms and the Monge-Ampère equations, J. Diff. Geom., 46 (1997), 335-373

Henri Anciaux, Universidade de São Paulo, ime, Bloco A, 1010 Rua do Matão, Cidade Universitária, 05508-090 SÃo Paulo, BRAZIL,

E-mail address: henri.anciaux@gmail.com

Departamento de Matemáticas, Universidad De JaÉn, 23071 JaÉn, SPAIN

E-mail address: icastro@ujaen.es 